\title{
In Vivo and In Vitro Elution of Analgesics from Multilayered Poly(D,L)-lactide-co-glycolide Nanofibers Incorporated Ureteral Stents
}

\author{
Yi-Chia Lin, ${ }^{1}$ Kuo-Sheng Liu, ${ }^{2}$ Demei Lee, ${ }^{3}$ Min-Jhan Li, ${ }^{3}$ \\ Shih-Jung Liu $\mathbb{D}^{3,4}$ and Hiroshi Ito ${ }^{5}$ \\ ${ }^{1}$ Department of Urology, Shin Kong Wu Ho-Su Memorial Hospital, Taipei 111, Taiwan \\ ${ }^{2}$ Department of Cardiovascular Surgery, Chang Gung Memorial Hospital, Linkou, Taoyuan 333, Taiwan \\ ${ }^{3}$ Department of Mechanical Engineering, Chang Gung University, Taoyuan 333, Taiwan \\ ${ }^{4}$ Department of Orthopedic Surgery, Chang Gung Memorial Hospital, Linkou, Taoyuan 333, Taiwan \\ ${ }^{5}$ Research Center for Green Materials and Advanced Processing, Yamagata University, Yamagata 992-8510, Japan
}

Correspondence should be addressed to Shih-Jung Liu; shihjung@mail.cgu.edu.tw

Received 20 November 2017; Accepted 29 January 2018; Published 7 March 2018

Academic Editor: Nader Shehata

Copyright (C) 2018 Yi-Chia Lin et al. This is an open access article distributed under the Creative Commons Attribution License, which permits unrestricted use, distribution, and reproduction in any medium, provided the original work is properly cited.

\begin{abstract}
We develop novel analgesic-eluting nanofiber-incorporated ureteral stents that offer sustained release of lidocaine and ketorolac for local drug delivery. Lidocaine and poly(D,L)-lactide-co-glycolide (PLGA) were dissolved in hexafluoroisopropanol and were electrospun into nonwoven nanofibers onto the surface of ureteral stents. This was followed by electrospinning of another layer of PLGA nanofibers containing ketorolac. Electrospun drug-loaded nanofibers were then characterized using scanning electron microscopy, Fourier transform infrared spectroscopy, and water contact angle analysis. In addition, the elution behavior characteristics of the analgesics, both in vivo and in vitro, from the nanofiber-incorporated stents were evaluated. Experimental results indicate that the analgesic-eluting ureteral stents could liberate high strengths of analgesics in vitro and in vivo for at least 50 and 30 days, respectively. The analgesic-eluting nanofiber-incorporated ureteral stents are potentially applicable for alleviating the discomfort associated with stent implant.
\end{abstract}

\section{Introduction}

Ureteral stents have been used to reestablish or maintain the ureter patency and are one of the most common devices used by urologists $[1,2]$. For those requiring short-term stenting, pain and discomfort caused by the device are the most common complaints, especially during activity and urination. According to the literature, stent-related pain has been reported by more than $80 \%$ of patients [3].

Stent pain pathophysiology is influenced by several factors and can likely be the result of mucosal irritation together with retrograde reflux of urine. Even though there is no consensus on the relationship between stent length/diameter and stent-related pain, stent size should be optimized to avoid potential dislodgement. A wide variety of medications have been administered to treat ureteral stent discomfort.
Alpha-adrenergic blockers have been shown to be efficacious and should be used together with anticholinergics and nonsteroidal anti-inflammatory drugs (NSAIDs) in treating ureteral stent-associated pain. Clinically, the combined use of lidocaine and ketorolac has been widely administered for postoperative pain release. Nevertheless, a few studies comparing these oral agents have shown minimal efficacy and a number of significant side effects in the treatment of ureteral stent discomfort [3].

In this study, we developed novel biodegradable lidocaine- and ketorolac-eluting nanofiber-incorporated ureteral stents for the local and sustainable delivery of analgesics. Lidocaine and poly(D,L)-lactide-co-glycolide (PLGA) were dissolved in hexafluoroisopropanol and then electrospun into nanofibers onto the surface of ureteral stents. This was followed by electrospinning of another layer of PLGA 


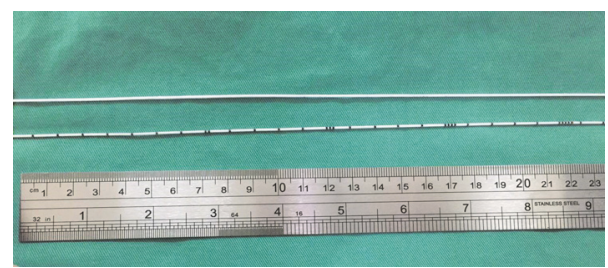

FIGURE 1: Ureteral stents (top: loaded with nanofibers; bottom: bare stent).

nanofibers containing ketorolac. Electrospun nanofibers were observed using scanning electron microscopy (SEM) for morphological analysis. The in vitro elution characteristics of the analgesics from the nanofibrous mat were explored. Furthermore, a rabbit model was used to investigate the delivery of analgesics to the urine and blood.

\section{Materials and Method}

2.1. Manufacture of Analgesic-Eluting Stents. The polymeric material was PLGA 50:50 (Sigma-Aldrich, St. Louis, MO, USA). Lidocaine hydrochloride, ketorolac, and hexafluoroisopropanol were also purchased from Sigma-Aldrich.

By employing the electrospinning technique, biodegradable analgesic-eluting nanofibers were electrospun to coat the external surface of a Double-J ureteral stent (Model BT-DJ7024-110, 7 Fr $\times 24$ cm; Bioteq Inc., Taiwan). Lidocaine and PLGA (40 mg : $240 \mathrm{mg}$ ) were dissolved in $1 \mathrm{~mL}$ hexafluoroisopropanol. The solution was then electrospun into nonwoven nanofibers onto the surface of a ureteral stent mounted on a motor. This was followed by electrospinning of a layer of PLGA/ketorolac (240 mg: $40 \mathrm{mg}$ ) nanofibers onto the stent. The motor was set at $300 \mathrm{rpm}$. The distance between the needle tip and the stent was $15 \mathrm{~cm}$, and a positive voltage of $20 \mathrm{kV}$ was applied while the solution was delivered at $3.6 \mathrm{~mL} / \mathrm{hr}$. Figure 1 shows the analgesic-eluting nanofiberloaded Double-J stent and the bare stent. Their external diameters were 2.55 and $2.20 \mathrm{~mm}$, respectively. All stents mounted with a nanofibrous matrix were held in a vacuum oven at $40^{\circ} \mathrm{C}$ for three days to evaporate residual solvent.

2.2. Evaluation of Electrospun Nanofibrous Matrix. Electrospun nanofibers were observed under a scanning electron microscope (S-3000N; Hitachi, Japan) for morphological analysis. To analyze diameter distribution, fifty nanofibers were stochastically selected from the SEM photos and characterized. Additionally, the porosity of the nanofibers was calculated as

$$
\text { Pore }(\%)=\left\{1-\frac{\rho_{\text {membrane }}}{\rho_{\text {polymer }}}\right\} \text {, }
$$

where $\rho_{\text {membrane }}$ and $\rho_{\text {polymer }}$ indicate the densities of the nanofibers and the polymer, respectively.

Infrared spectra of the analgesic-loaded nanofibrous matrix were evaluated employing Fourier transform infrared (FTIR) spectrometry. Experiments were conducted on a Nicolet iS5 spectrometer (Thermo Fisher Scientific, Waltham,

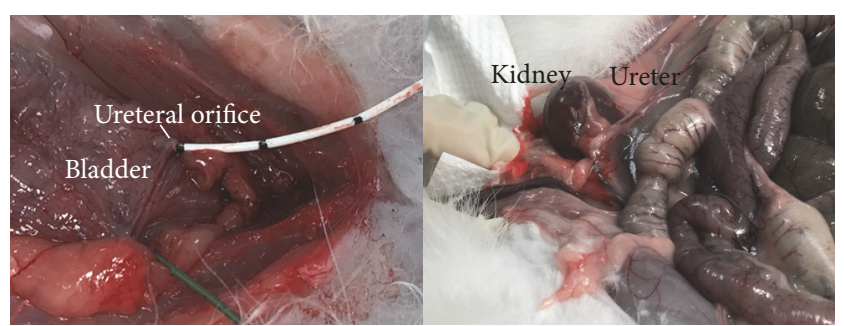

Figure 2: Deployment of analgesic-eluting ureteral stents in vivo.

MA, USA) at a resolution of $4 \mathrm{~cm}^{-1}$ and 32 scans over the $400-4000 \mathrm{~cm}^{-1}$ range.

The wetting properties of the nanofibrous mats were characterized by measuring the contact angle of small droplets of liquid on the membranes. Water droplets were applied to the membrane surface using a syringe. Computer software (First Ten Angstroms, USA) was used to quantify a droplet image from points marked along the droplet-air interface to calculate the contact angle at the droplet-membrane surface interface $(N=3)$.

2.3. Characterization of In Vitro Drug Elution. The elution behavior characteristics of lidocaine and ketorolac from the analgesic-eluting ureteral stents were evaluated using an in vitro release scheme. Three $2 \mathrm{~cm}$ long specimens were cut from the lidocaine/ketorolac-loaded stents, and each was put into a plastic test tube containing $1 \mathrm{~mL}$ phosphate buffer solution and then held at $37^{\circ} \mathrm{C}$ for 24 hours. Each day for 50 days, the solution was replaced with fresh phosphate buffer solution, and the analgesic concentrations were quantified using high-performance liquid chromatography.

2.4. Animal Study. Five New Zealand white rabbits weighing $2.8-3.3 \mathrm{~kg}$ were used for animal studies. All animal procedures were approved by the Institutional Animal Care and Use Committee. All rabbits were anesthetized using isoflurane inhalation through a vaporizer (Matrix, USA) in a plastic box $(40 \mathrm{~cm} \times 20 \mathrm{~cm} \times 28 \mathrm{~cm})$. General anesthesia was maintained during the entire surgical procedures by way of mask inhalation of isoflurane. After standard skin disinfection and aseptic dressing, lower midline laparotomy was performed. The urinary bladder was identified and a horizontal cystotomy was performed. A 7 Fr ureteral catheter was used to catheterize the ureter, and its position was verified through intraoperative ultrasound and urine aspiration. A 0.035-inch guidewire was inserted through the catheter into the renal pelvis. The catheter was removed, and a $5 \mathrm{~cm}$ segment of the analgesic-eluting nanofiber-incorporated ureter stent was inserted over the guidewire into the ureter (Figure 2). The cystotomy and laparotomy were then closed with absorbable sutures. After surgeries, all animals were allowed to move freely in individual cages and given standard rabbit chow and sterilized drinking water ad libitum. The temperature was maintained at $24^{\circ} \mathrm{C}$, while the humidity was set at $70 \%$. Urine and blood samples were collected 1, 3, 7, 14, 21, and 28 days after implantation for drug concentration analysis. Urine was gathered via ultrasound-guided sterile suprapubic aspiration, 

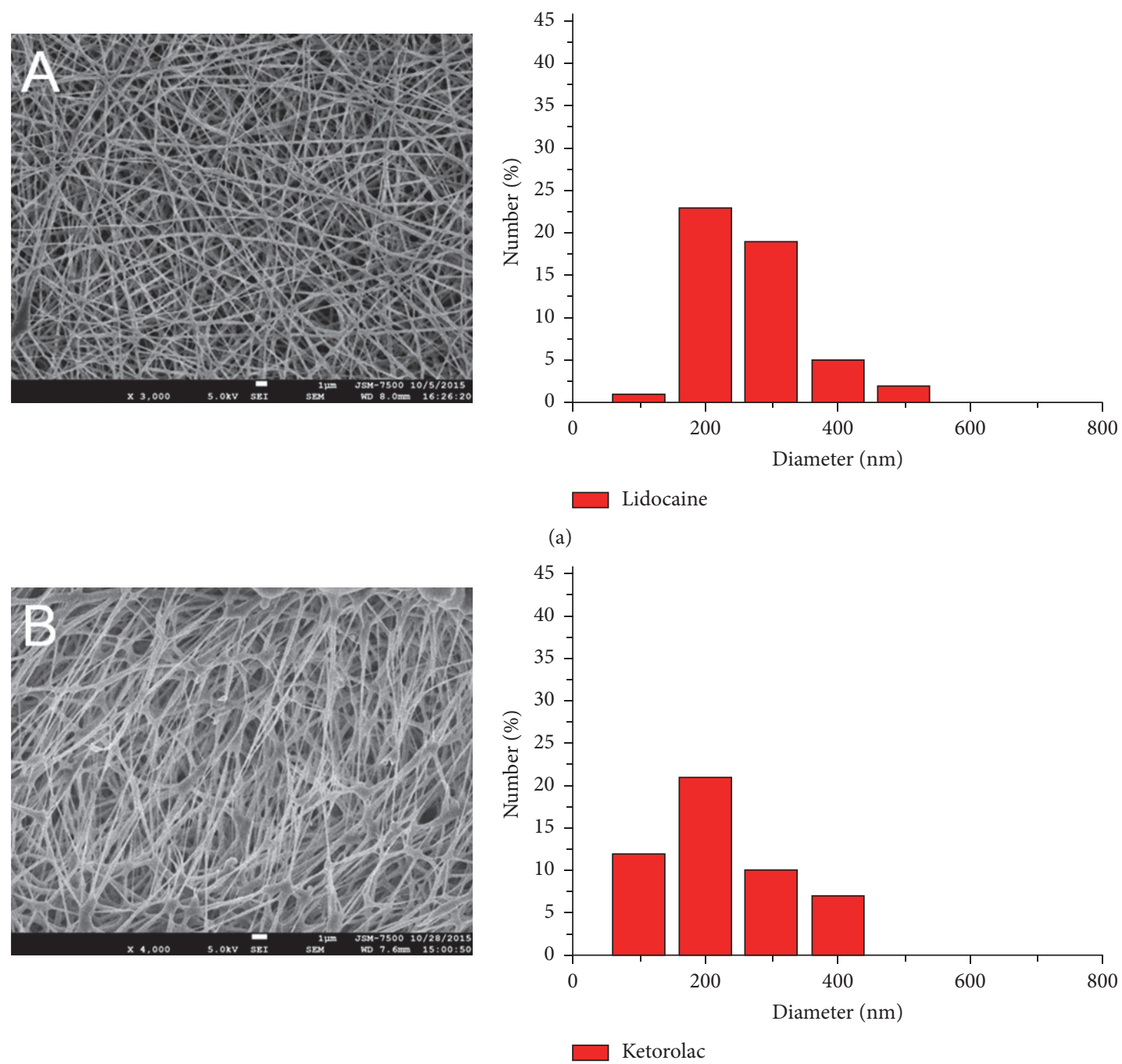

(b)

FIGURE 3: Fiber diameter distribution of (a) lidocaine-loaded and (b) ketorolac-loaded nanofibers as seen using scanning electron microscopy.

while blood samples were gathered into syringes via puncture of the marginal ear vein.

2.5. Statistical Analysis. Collected data were analyzed using analysis of variance (ANOVA) obtained through commercially available software (SPSS Version 12.0; SPSS Inc., Chicago, IL, USA). Means and standard deviations were calculated for each variable. Significance was recognized when $p$ was less than 0.05 .

\section{Results}

3.1. Evaluation of Fabricated Analgesic-Eluting Nanofibers. Figure 3 shows the SEM images of the analgesic-eluting nanofibrous mat as well as the fiber diameter distributions. The computed mean ( \pm standard deviation) diameters for the lidocaine- and ketorolac-loaded nanofibers were $278.4 \pm$ $39.4 \mathrm{~nm}$ and $182.8 \pm 50.5 \mathrm{~nm}$, respectively $(p<0.05)$. The porosity of the nanofibers was high $(89.2 \% \pm 5.2 \%$ and 93.5\% $\pm 4.7 \%$, resp.; $p<0.05$ ).
Figure 4 illustrates the FTIR spectra of PLGA mats without and with added analgesics. In the latter, an additional absorption peak, at $3400 \mathrm{~cm}^{-1}$, is attributable to the $\mathrm{N}-\mathrm{H}$ bonds of ketorolac and lidocaine. Meanwhile, the absorption at $1725 \mathrm{~cm}^{-1}$ corresponds to the $\mathrm{C}=\mathrm{O}$ bonds, mainly from the addition of the analgesics. Furthermore, the absorbance peak near $1300 \mathrm{~cm}^{-1}$ could be from enhancement in the $\mathrm{C}$-O bonds in the analgesics $[4,5]$. The spectra resolution confirms the successful embedment of analgesics in the PLGA matrix.

Figure 5 shows the measured water contact angles. For pure PLGA nanofibers, ketorolac-loaded nanofibers, and lidocaine-loaded nanofibers, the angles were $124.0^{\circ}, 118.3^{\circ}$, and $74.5^{\circ}$, respectively. The addition of analgesics clearly improved the hydrophilic characteristics of PLGA nanofibers. Additionally, lidocaine-incorporated nanofibrous mats showed superior hydrophilicity over ketorolac-incorporated mats.

3.2. Elution of Lidocaine and Ketorolac from Analgesic-Eluting Stents In Vitro. Figures 6(a) and 6(b) plot the in vitro 


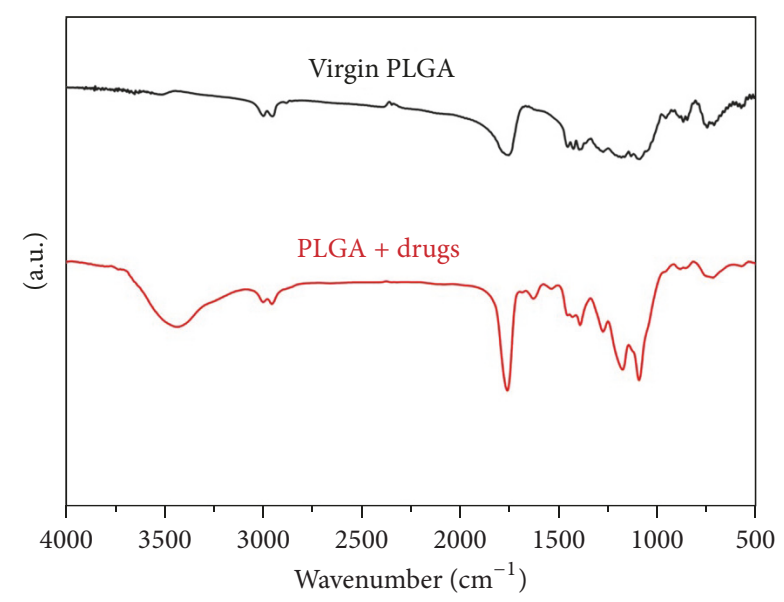

FIGURE 4: Fourier transform infrared spectra of electrospun poly(D,L)-lactide-co-glycolide (PLGA) and analgesic-loaded PLGA nanofibers.

daily and accumulated elution behaviors of the analgesics. Based on these figures, the drug-eluting stents continuously liberated high strengths of analgesics for over the 50-day period. Lidocaine release was characterized by an initial burst for five days followed by a nearly linear release from days 6 to 30 . Thereafter, the drug concentration gradually decreased. The elution of ketorolac, on the other hand, showed a tiny fast release over 1-3 days and then a steady diminishing release for the following days. Only $70 \%$ of the loaded ketorolac was released by day 50 .

3.3. In Vivo Release Characteristics. Figure 7(a) illustrates the lidocaine concentrations in urine and in blood at various days, while Figure 7(b) illustrates the drug levels of ketorolac. The analgesic-eluting ureteral stents could offer sustainable elution of lidocaine and ketorolac for more than 30 days in vivo. On the contrary, the blood levels were much lower throughout the study period.

\section{Discussion}

Ureteral stents have been widely used to relieve ureteral obstruction from edema caused by endoscopic manipulation or intracorporeal lithotripsy. They play important roles in a wide range of situations where urinary drainage is necessary. Despite their clinical usefulness, pain over the flank, low abdomen, groin, and external genitalia is common after insertion of a ureteral stent. This is typically managed using oral NSAIDs or other analgesic medications [3]. However, when given systemically, these pharmaceuticals result in adverse effects such as pruritus, vomiting, nausea, dizziness, and sedation. Specifically, NSAIDs can create renal function impairment and gastrointestinal bleeding [6].

Ureteral stents can be incorporated with drugs and other materials to assist patient comfort and/or to forestall encrustation. Drug-loaded stents liberate medications that perform locally on the bladder to reduce irritation and pain $[7,8]$. To date, various approaches have been employed in an attempt to do so and have focused on developing novel surface coatings and drug-elution strategies. Krambeck et al. [3] examined the short-term safety and efficacy of a ketorolacincorporated ureteral stent and confirmed its safety. They also found that young male patients in particular appeared to require less pain medication when the ketorolac-loaded stent was used. Pechey et al. [9] investigated methoxy polyethylene glycol-dihydroxyphenylalanine-coated ureteral stents in a rabbit model and found that they strongly resist bacterial attachment, resulting in improved infection clearance over that of uncoated devices. Cadieux et al. [10] explored the effects of triclosan-loaded stent segments on the growth and survival of Proteus mirabilis and found that triclosanloaded stents greatly reduce $P$. mirabilis growth and survival compared to controls in a rabbit UTI model.

We have successfully fabricated analgesic-eluting nanofiber-incorporated ureteral stents via electrospinning. A drug-loaded nanofibrous matrix could offer sustained elution of analgesics for over 50 days in vitro. After fabrication, while most of the drug was embedded within the volume of the polymeric membranes, a certain amount of drug could have been deposited on the exterior of the nanofibrous matrix, subsequently causing the initial burst release. Following the initial burst, the release of pharmaceuticals was dominated by polymeric material degradation [11]. The nanofibrous matrix therefore displayed a fairly stable release of lidocaine and ketorolac for more than 50 days.

After stent implantation, most of the drug incorporated with the nanofibers will be washed away by urine. Some will enter the bloodstream after release [6]. Our experimental results suggest that drug concentrations in the urine are much higher than in the blood. This provides advantages in terms of delivering high drug concentrations at the target site with minimal systemic side effects. Additionally, the in vivo release of lidocaine did not show the initial burst release. This might be explained by the static nature of the in vitro test, where the eluent was replaced every 24 hours. Relatively, the frequency of urine release in vivo by the animals is greater than what we used in vitro. The initial burst release would therefore be minimized. On the other hand, lidocaine was found to be released faster than ketorolac. This might be because lidocaine exhibits greater hydrophilicity. It thus becomes easier for lidocaine to be diffused by the surrounding fluids. Meanwhile, lidocaine possesses a less molecular weight (lidocaine: $234 \mathrm{~g} / \mathrm{mol}$; ketorolac: $364 \mathrm{~g} / \mathrm{mol}$ ) [6]. The release rate of lidocaine increased accordingly. Furthermore, even though the lidocaine concentration was much lower in the plasma than in the urine, it exceeded the toxic concentration (i.e., $>6 \mu \mathrm{g} / \mathrm{mL}$ ) [11]. Fortunately, all rabbits survived throughout the study period. A more elaborate study will be required to determine the appropriate drug dosage (or the appropriate size of the nanofibers) to achieve effective analgesia at the local area while maintaining a subtoxic level in the plasma.

Biodegradable polymers used for drug delivery must have the following properties [12]: (1) evoking a minimum inflammatory response, (2) possessing a controllable degradation time, (3) exhibiting appropriate mechanical properties, (4) producing nontoxic products during degradation, and (5) 


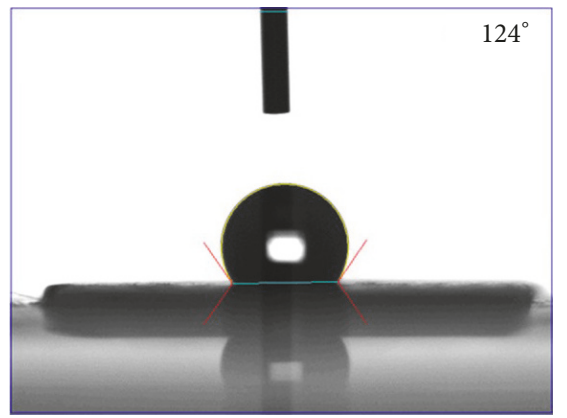

(a)

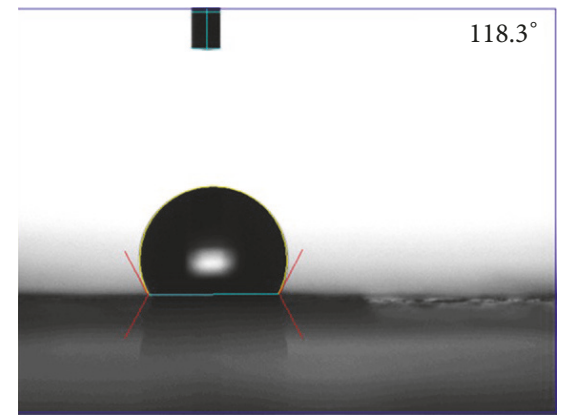

(b)

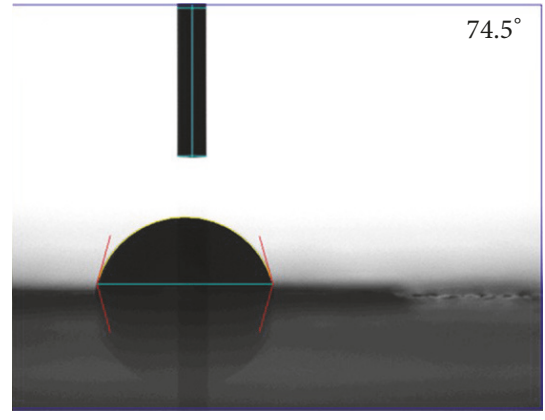

(c)

FIGURE 5: Water contact angles for (a) poly(D,L)-lactide-co-glycolide (PLGA), (b) PLGA/ketorolac, and (c) PLGA/lidocaine nanofibers.

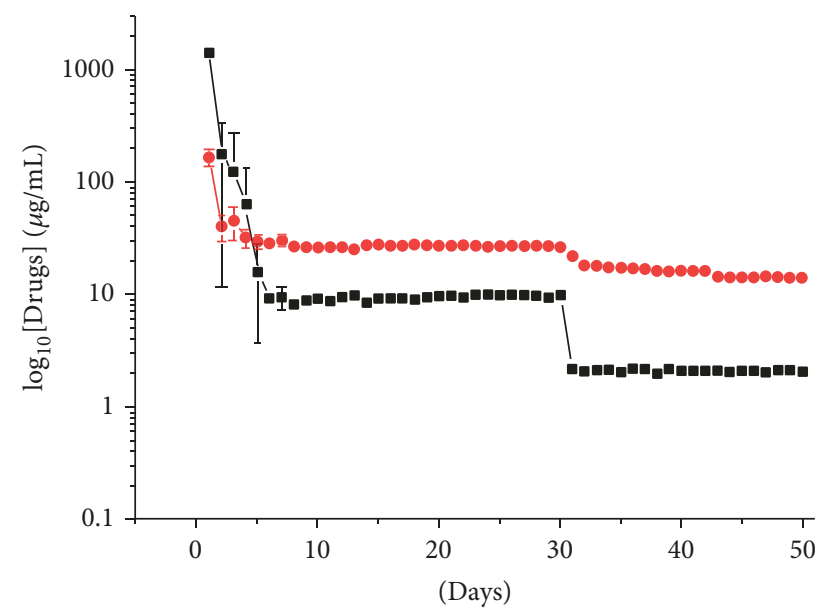

-- Lidocaine

-•- Ketorolac

(a)

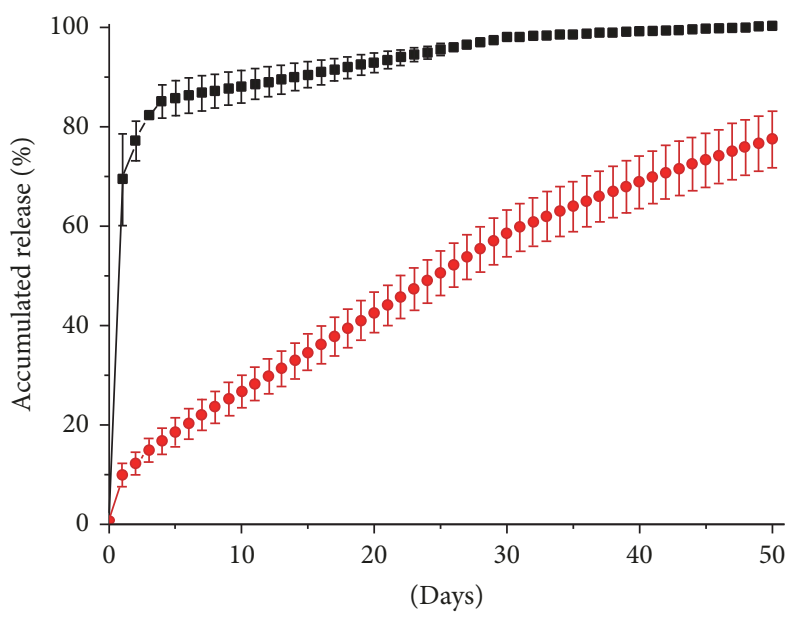

-- Lidocaine

- - Ketorolac

(b)

FIGURE 6: In vitro (a) daily and (b) accumulated release behaviors of analgesic-eluting stents.

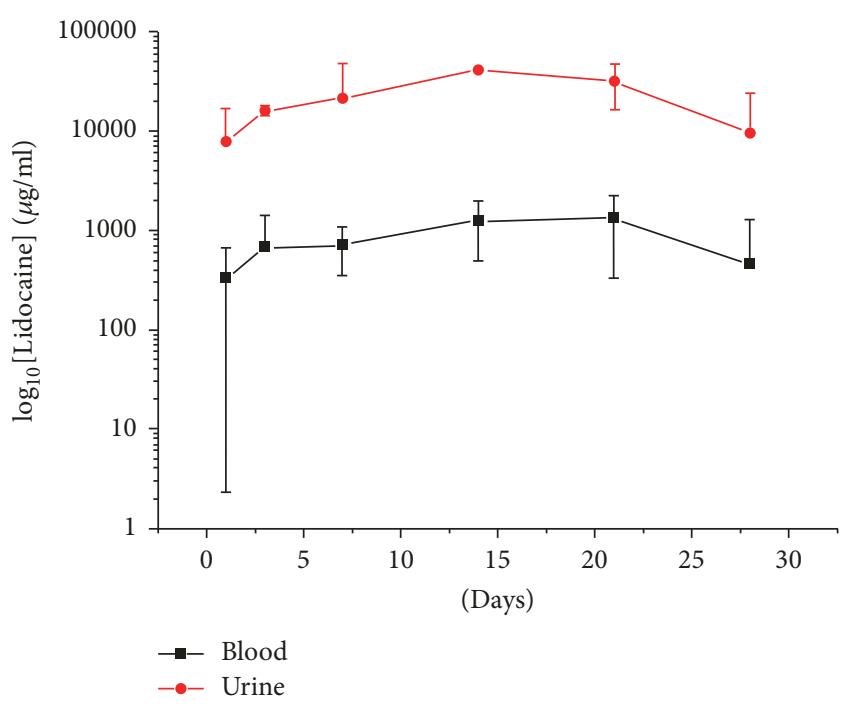

(a)

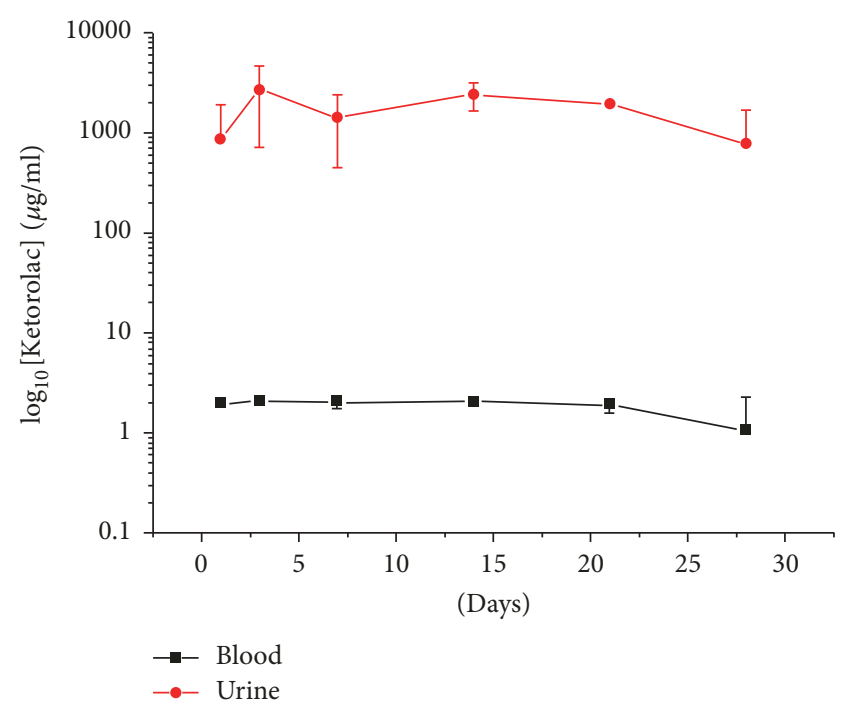

(b)

FIGURE 7: In vivo release behaviors of lidocaine and ketorolac from analgesic-eluting stents. 
possessing appropriate processing ability for the designated application. Among various biodegradable polymers, PLGA is one of the most investigated degradable polymers for biomedical applications. It belongs to a class of hydrolytically degradable polymers and has been approved by the U.S. Food and Drug Administration for human use in applications such as sutures and drug delivery devices. The material evokes a minimal inflammatory response and degrades through the hydrolysis of its ester linkages to generate biocompatible lactic and glycolic acids. The degradation time of PLGA is dependent primarily on the monomer ratio in production. For example, 50:50 PLGA, 75:25 PLGA, and 85:15 PLGA reportedly completely degrade within 1-2 months, 45 months, and 5-6 months [13], respectively. The relatively short degradation time of 50:50 PLGA makes it an excellent material as a vehicle for drug delivery. Electrospinning, on the other hand, has been recognized as a simple and versatile method for fabrication of polymer nanofibers. Because of their internal architecture, nanofibers are well-suited for various medicinal applications [14]. The inherently high surface-to-volume ratio of electrospun nanofibers promotes cell attachment [15-17] and facilitates mass transfer and effective drug release [18, 19]. By employing the electrospinning technology, we can manufacture nanofiber-loaded stents incorporated with different pharmaceuticals for various ureteral applications.

Finally, despite its contributions, this study possesses limitations. First, while our results show a local and sustainable elution of ketorolac and lidocaine from the nanofibrous matrix-loaded ureteral stents, the efficacy of released analgesics is yet to be confirmed. Further investigation of the analgesic-loaded ureteral stents in an animal pain model is required to address this question. Second, the animal model used in evaluating the expression of drug-incorporated stents is limited in terms of its ability to be reproduced in human studies. In addition, the use of pig model will be a better model for the study of ureteral stents in vivo. All these are potential topics for our future studies.

\section{Conclusions}

We have successfully fabricated biodegradable analgesiceluting nanofibers from PLGA polymers via electrospinning for ureteral stent-associated applications and shown the elution behaviors of two analgesics from the nanofibrous matrix in vitro. Nanofibers can provide sustained release of lidocaine and ketorolac in vitro for 50 days. Furthermore, animal (rabbit) experiments using the analgesic-eluting stents demonstrate that the nanofibrous mats could offer in vivo release of lidocaine and ketorolac at high levels over a 30day period. The drug-eluting stents we developed and used in this study show that analgesics can be sustainably transported to local target sites. This novel drug-eluting stent has the potential to be used to treat pain associated with ureteral stent placement.

\section{Conflicts of Interest}

All authors declare no conflicts of interest.

\section{Acknowledgments}

Chang Gung Memorial Hospital (Contract no. CMRPD2H0031) and the Ministry of Science and Technology, Taiwan (Contract no. 104-2221-E-182-048-MY3), financially supported this research.

\section{References}

[1] C. Koprowski, C. Kim, P. K. Modi, and S. E. Elsamra, "Ureteral stent-associated pain: A review," Journal of Endourology, vol. 30, no. 7, pp. 744-753, 2016.

[2] R. Miyaoka and M. Monga, "Ureteral stent discomfort: etiology and management," Indian Journal of Urology, vol. 25, no. 4, pp. 455-460, 2009.

[3] A. E. Krambeck, R. S. Walsh, J. D. Denstedt et al., "A novel drug eluting ureteral stent: a prospective, randomized, multicenter clinical trial to evaluate the safety and effectiveness of a ketorolac loaded ureteral stent," The Journal of Urology, vol. 183, no. 3, pp. 1037-1043, 2010.

[4] L. Fernandes Fraceto, L. De Matos Alves Pinto, L. Franzoni et al., "Spectroscopic evidence for a preferential location of lidocaine inside phospholipid bilayers," Biophysical Chemistry, vol. 99, no. 3, pp. 229-243, 2002.

[5] A. P. Rokhade, S. A. Agnihotri, S. A. Patil, N. N. Mallikarjuna, P. V. Kulkarni, and T. M. Aminabhavi, "Semi-interpenetrating polymer network microspheres of gelatin and sodium carboxymethyl cellulose for controlled release of ketorolac tromethamine," Carbohydrate Polymers, vol. 65, no. 3, pp. 243-252, 2006.

[6] C.-W. Kao, D. Lee, M.-H. Wu, J.-K. Chen, H.-L. He, and S.J. Liu, "Lidocaine/ketorolac-loaded biodegradable nanofibrous anti-adhesive membranes that offer sustained pain relief for surgical wounds," International Journal of Nanomedicine, vol. 12, pp. 5893-5901, 2017.

[7] L. Yang, S. Whiteside, P. A. Cadieux, and J. D. Denstedt, "Ureteral stent technology: drug-eluting stents and stent coatings," Asian Journal of Urology, vol. 2, no. 4, pp. 194-201, 2015.

[8] D. Lange, C. N. Elwood, and B. H. Chew, "Biomaterials in urology - Beyond drug eluting and degradable - A rational approach to ureteral stent design," in Biomaterials - Physics and Chemistry, R. Pignatello, Ed., 2011.

[9] A. Pechey, C. N. Elwood, G. R. Wignall et al., "Anti-Adhesive Coating and Clearance of Device Associated Uropathogenic Escherichia coli Cystitis," The Journal of Urology, vol. 182, no. 4, pp. 1628-1636, 2009.

[10] P. A. Cadieux, B. H. Chew, B. E. Knudsen et al., "Triclosan loaded ureteral stents decrease Proteus mirabilis 296 infection in a rabbit urinary tract infection model," The Journal of Urology, vol. 175, no. 6, pp. 2331-2335, 2006.

[11] Y.-Y. Tseng, J.-Y. Liao, W.-A. Chen, Y.-C. Kao, and S.-J. Liu, "Biodegradable poly([D,L]-lactide-co-glycolide) nanofibers for the sustainable delivery of lidocaine into the epidural space after laminectomy," Nanomedicine, vol. 9, no. 1, pp. 77-87, 2014.

[12] B. D. Ulery, L. S. Nair, and C. T. Laurencin, "Biomedical applications of biodegradable polymers," Journal of Polymer Science Part B: Polymer Physics, vol. 49, no. 12, pp. 832-864, 2011.

[13] J. C. Middleton and A. J. Tipton, "Synthetic biodegradable polymers as orthopedic devices," Biomaterials, vol. 21, no. 23, pp. 2335-2346, 2000. 
[14] R. Vasita and D. S. Katti, "Nanofibers and their applications in tissue engineering," International Journal of Nanomedicine, vol. 1, no. 1, pp. 15-30, 2006.

[15] R. L. Dahlin, F. K. Kasper, and A. G. Mikos, "Polymeric nanofibers in tissue engineering," Tissue Engineering - Part B: Reviews, vol. 17, no. 5, pp. 349-364, 2011.

[16] N. G. Rim, C. S. Shin, and H. Shin, "Current approaches to electrospun nanofibers for tissue engineering," Biomedical Materials, vol. 8, no. 1, Article ID 014102, 2013.

[17] H. S. Yoo, T. G. Kim, and T. G. Park, "Surface-functionalized electrospun nanofibers for tissue engineering and drug delivery," Advanced Drug Delivery Reviews, vol. 61, no. 12, pp. 10331042, 2009.

[18] J. Hrib, J. Sirc, R. Hobzova et al., "Nanofibers for drug delivery - Incorporation and release of model molecules, influence of molecular weight and polymer structure," Beilstein Journal of Nanotechnology, vol. 6, no. 1, pp. 1939-1945, 2015.

[19] X. Hu, S. Liu, G. Zhou, Y. Huang, Z. Xie, and X. Jing, "Electrospinning of polymeric nanofibers for drug delivery applications," Journal of Controlled Release, vol. 185, no. 1, pp. 12-21, 2014. 


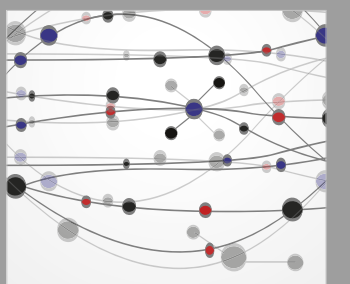

The Scientific World Journal
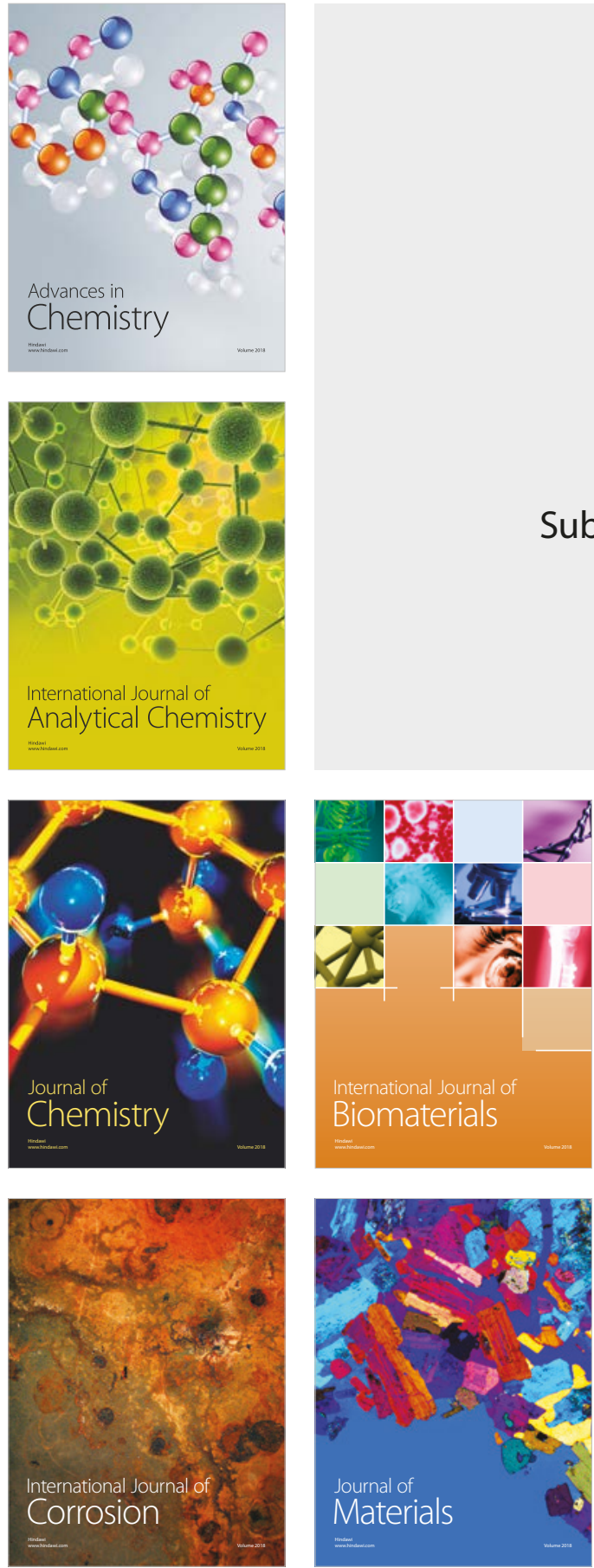

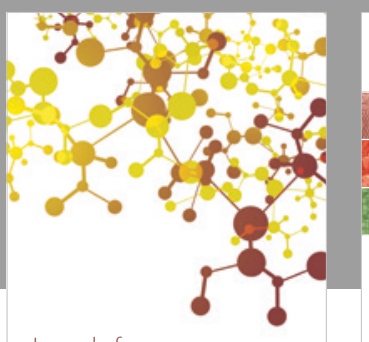

Journal of

Applied Chemistry
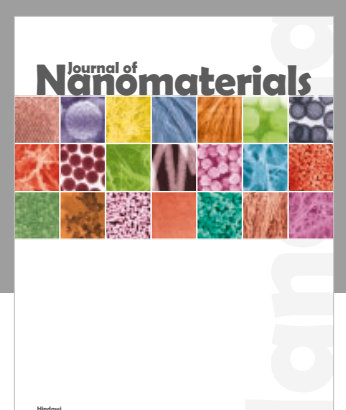

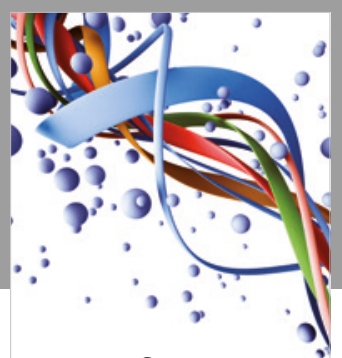

Scientifica

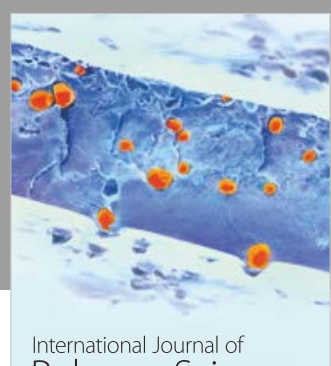

Polymer Science

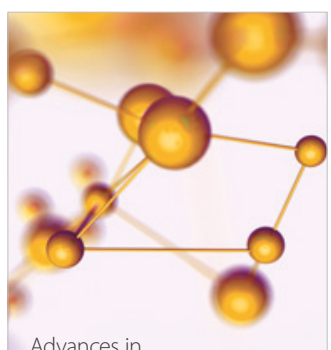

Physical Chemistry
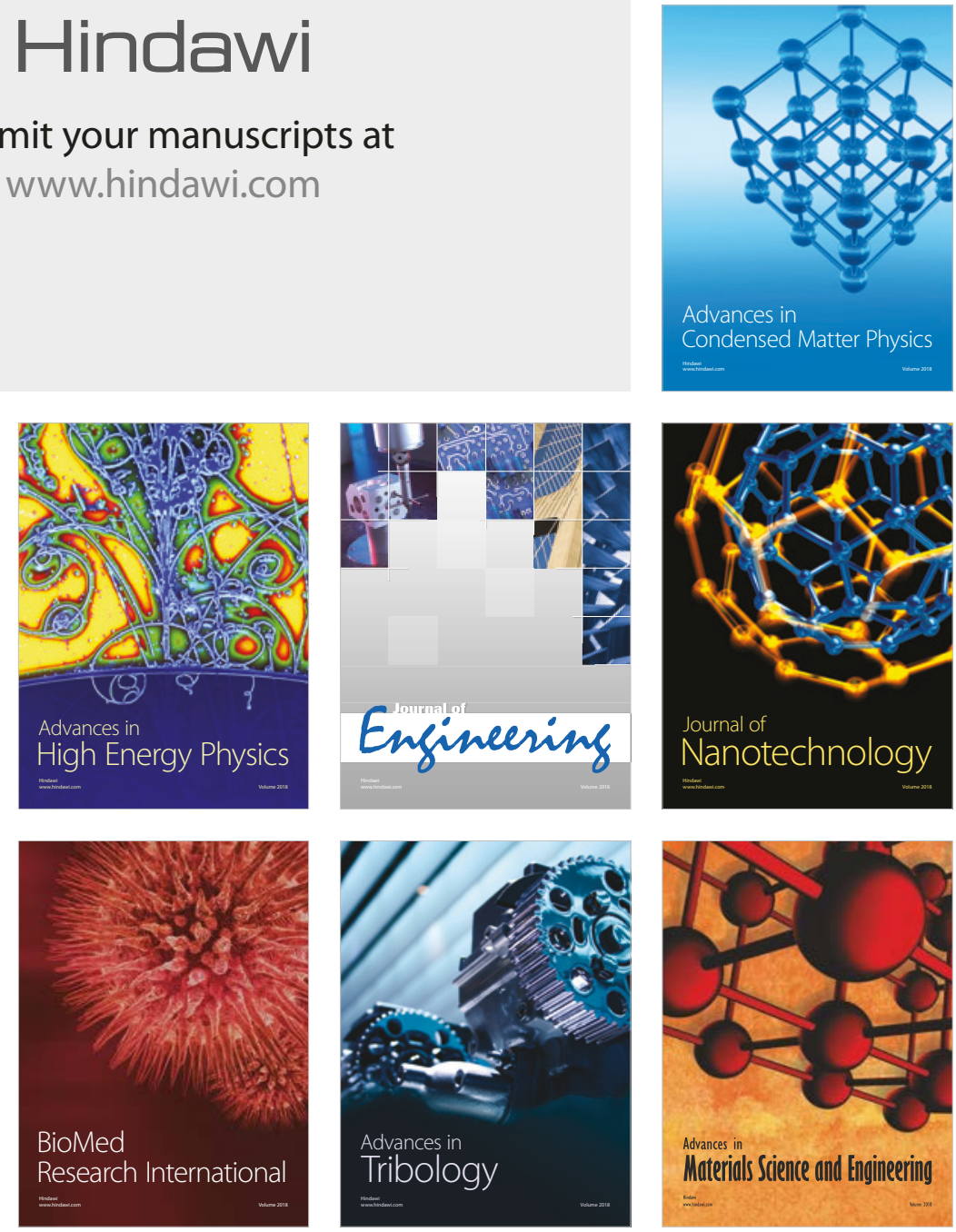